\title{
Microscopic Calculations of Collective Flow Probing the Short-Range Nature of the Nuclear Force
}

\author{
J. J. Molitoris, J. B. Hoffer, ${ }^{(a)}$ H. Kruse, ${ }^{(b)}$ and H. Stöcker \\ Department of Physics and Astronomy and National Superconducting Cyclotron Laboratory, Michigan State University, \\ East Lansing, Michigan 48824 \\ (Received 27 April 1984)
}

\begin{abstract}
Collisions between two nuclei have been modeled by numerical solution of classical approximations to the equations of motion of the constituent nucleons. For the reaction $\mathrm{Nb}(400 \mathrm{MeV} / \mathrm{u})+\mathrm{Nb}$, a correlated sidewards emission of nucleons is observed. This is attributed to the repulsive short-range component of the nucleon-nucleon potential. A strong dependence of the flow angle on the impact parameter is observed, in accord with recent experimental results.
\end{abstract}

PACS numbers: $25.70 . \mathrm{Np}$

The recent experimental observation ${ }^{1}$ of sidewards peaks in the emission pattern of fragments emitted in collisions of heavy nuclear systems has stimulated a dispute among theorists about how to interpret these data. It has been shown ${ }^{2}$ that the observations are in agreement with the results of macroscopic nuclear fluid dynamical calculations, but several microscopic calculations done to reproduce the sidewards emission [via the intranuclear cascade (INC) approach ${ }^{3,4}$ ] failed - the angular distributions obtained were always forward peaked. ${ }^{1,2}$

We have recently developed a many-body equations-of-motion (EOM) approach to study heavy-ion collisons. Our approach is analogous to that of Bodmer, Panos, and MacKellar ${ }^{5}$ and Wilets and co-workers. ${ }^{6}$ Hamilton's equations of motion are solved for an ensemble of $A$ nucleons with simultaneous mutual two-body interactions between all particles:

$$
\dot{\overrightarrow{\mathrm{p}}}_{i}=-\vec{\nabla}_{r_{i}} H, \quad \dot{\overrightarrow{\mathrm{r}}}_{i}=\vec{\nabla}_{p_{i}} H \text {. }
$$

The Hamiltonian is

$$
H=\sum_{i=1}^{A} \frac{p_{i}^{2}}{2 m}+\sum_{i<j} V(r),
$$

where $r=\left|\overrightarrow{\mathrm{r}}_{i}-\overrightarrow{\mathrm{r}}_{j}\right|$ is the distance between nucleons $i$ and $j$.

This classical nonrelativistic approach neglects spin, the Pauli principle, and the uncertainty principle. On the other hand, this approach allows for a study of the simultaneous (classical) interactions between many particles in contrast to the independent-particle models such as the intranuclear cascade, which treat nuclear collisions as a simple superposition of successive free-space nucleon-nucleon collisions. Also, the EOM approach allows for a systematic study of the repulsive core of the nucleon-nucleon interaction (because of its deterministic nature, the EOM calculation pro- duces an excluded-volume effect), which is of interest in connection with the possible study of nuclear matter properties at high baryon densities.

We also neglect Coulomb forces and pion production, although the former are trivial to include and we have checked that one obtains identical flow results when Coulomb interaction is included at a typical impact parameter. At $E_{\text {c.m. }}=100 \mathrm{MeV} / \mathrm{u}$, the total Coulomb energy is only a small fraction of the incident kinetic energy. Pion production does not play a significant role for the present investigation; in fact, less than $\frac{1}{2}$ pion is produced on the average in the collision studied here. ${ }^{4}$

The nucleon-nucleon potential consists of two terms, an attractive long-range Yukawa interaction and a repulsive short-range core ${ }^{5,6}$ :

$$
V=\left[V_{R} \exp \left(-K_{R} r\right)-V_{A} \exp \left(-K_{A} r\right)\right] / r,
$$

where $V_{R}=2970 \mathrm{MeV}$-fm, $V_{A}=765 \mathrm{MeV}$-fm, $K_{R}$ $=2.66 \mathrm{fm}^{-1}$, and $K_{A}=1.75 \mathrm{fm}^{-1}$. The parameters in the potential were chosen in a compromise between reproducing in the EOM calculation the $n$ $p$ differential scattering cross section at large angles $\theta_{\text {c.m. }}=90^{\circ}$ (which influences the transverse momentum transfer the most) and at the same time giving reasonable nuclear radii and binding energies.

The differential cross section is hard to obtain in a classical calculation because of diffraction and exchange effects. A meaningful quantity to fit is the viscosity moment of the scattering cross section,

$$
\sigma_{v}=2 \pi \int_{0}^{\pi} \sigma(\theta) \sin ^{2} \theta d(\cos \theta),
$$

which determines the viscosity and thermal conductivity in a Boltzmann-equation approach.5, 6 The present potential gives values of $41.1,31.9,27.5$, and $24.6 \mathrm{mb}$ for $\sigma_{v}$ at $100,200,300$, and $400 \mathrm{MeV}$, respectively. This compares well with the experimental values of $39.2,26.5,25.0 \mathrm{mb}$, which have 
been calculated by Bodmer and Panos. ${ }^{5}$ We calculate $d \sigma / d \Omega\left(90^{\circ}\right)$ to be $2.0 \mathrm{mb}$ at $400 \mathrm{MeV}$, which is quite close to the experimental value of $1.5 \mathrm{mb}$. If we assume charge exchange to occur with $50 \%$ probability in $n-p$ reactions, then the measured differential cross section at $400 \mathrm{MeV}$ is well reproduced for large angles $40^{\circ} \leqslant \theta_{\text {c.m. }} \leqslant 140^{\circ}$.

In the EOM approach, nuclei are described as an ensemble of protons and neutrons initially distributed randomly throughout a sphere with the nuclear radius $R=1.2 A^{1 / 3} \mathrm{fm}$. However, the nuclei obtained are not stable: They tend to collapse and evaporate many nucleons when the classical equations of motion are integrated over a typical collision time. In the present approach, a metastable ground state has been obtained by allowing the nucleons to drift toward the configuration of minimum energy of the chosen nucleon-nucleon potential which is a crystalline structure. ${ }^{6}$ The characteristic interparticle distance corresponds to that of the potential minimum. This minimization is continued only until the interparticle distances begin to show structure and the nuclear density is still constant. This leads to nuclei sufficiently stable for a collision calculation to be meaningful rather than resulting in a disruption of the nuclei before a collision can actually take place. Our potential has a minimum of $-4.67 \mathrm{MeV}$ at $R=1.85 \mathrm{fm}$. The resulting crystalline ground-state configuration has an average binding energy of $-31 \mathrm{MeV} / \mathrm{u}$. The nu-

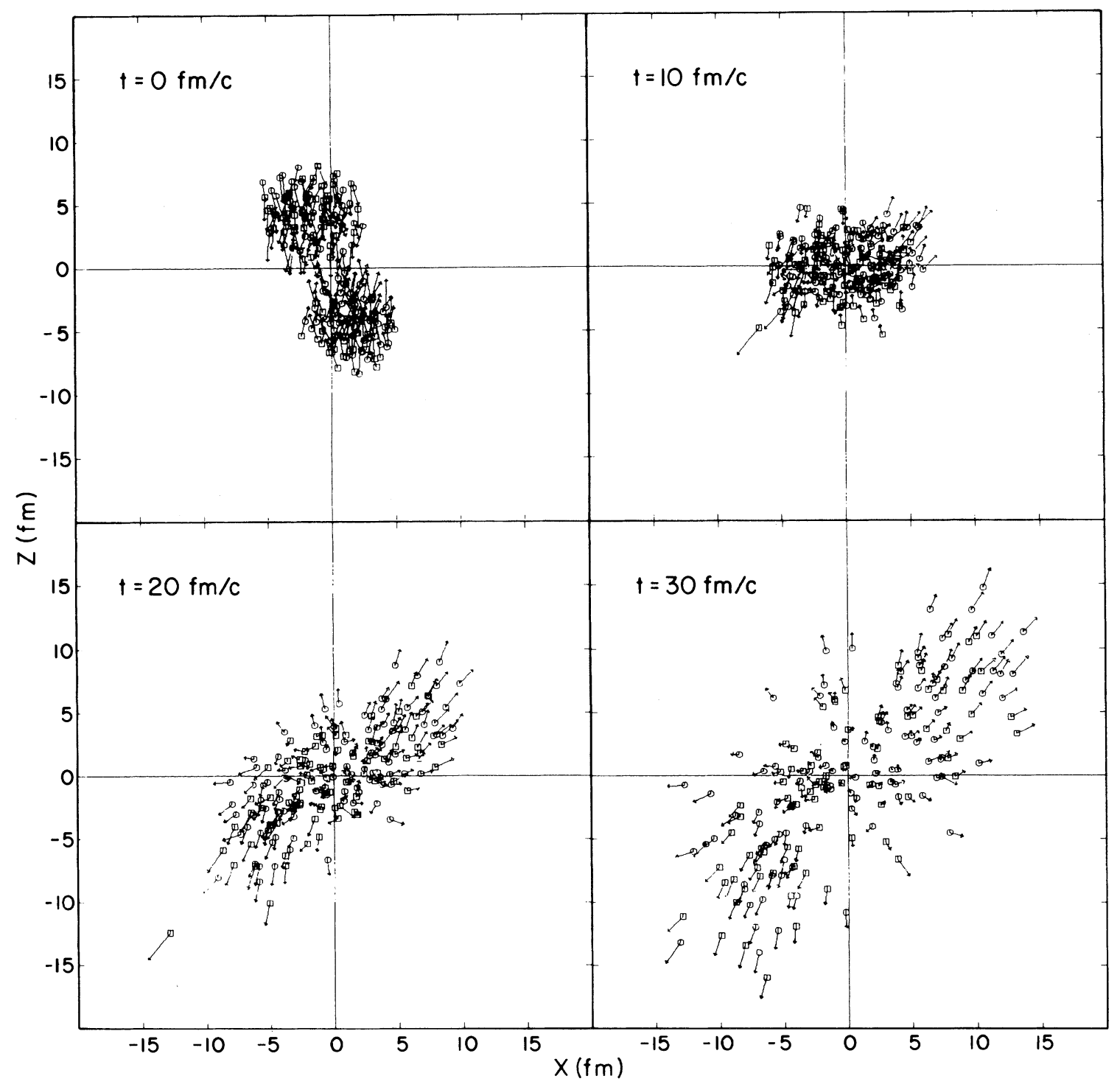

FIG. 1. Time evolution of a $\mathrm{Nb}(400 \mathrm{MeV} / \mathrm{u})+\mathrm{Nb}$ reaction at $b=3 \mathrm{fm}$. 
cleons then are given random Fermi momenta with an average Fermi energy of $23 \mathrm{MeV} / \mathrm{u}$ which results in an average binding energy of $7 \mathrm{MeV} / \mathrm{u}$. These nuclei are stable for $t=O(30 \mathrm{fm} / c)$, i.e., typical collision times.

To simulate a collision process numerically, the nuclei are Galilei-boosted with the respective center-of-mass momenta at given impact parameter. The equations of motion are integrated by a fourth-order Adams-Moulton predictor-corrector method. Energy conservation to better than 1\% has been demanded. A total of 535 collisions of $\mathrm{Nb}(400 \mathrm{MeV} / \mathrm{u})+\mathrm{Nb}$ have been performed, which provides reliable statistics for the global event-byevent analysis subsequently performed on the numerical "data." The computations are stopped after $t=30 \mathrm{fm} / c$, after which the flow results are found to be constant. The evolution of a collision at $b=3-\mathrm{fm}$ impact parameter is shown in Fig. 1 . The resulting sidewards flow can clearly be seen. Note that the average deflection angle of the center of mass of projectile and target nucleons, respectively, is approximately equal to the average calculated flow angle, thus associating a collective repulsion between the two nuclei.

The individual collisions are analyzed by diagonalizing the kinetic energy flow tensor, ${ }^{7}$

$$
F_{i j}=\sum_{v} p_{i}(v) p_{j}(v) / 2 m(v),
$$

where the sum is over all charged particles in a given event. By diagonalization of this tensor, the flow angle $\theta_{\mathrm{F}}$ is obtained for each event. The distribution of flow angles $d N / d \cos \theta_{\mathrm{F}}$ is presented in Fig. 2 for two impact-parameter intervals and compared to the experimental data. ${ }^{1}$ The qualitative and quantitative behavior of the flow pattern in the EOM model is very similar to the behavior observed in hydrodynamics ${ }^{2}$ : The flow angle $\theta_{\mathrm{F}}$ rises smoothly from $0^{\circ}$ at large impact parameters to $90^{\circ}$ at $b=0$. A finite range of impact parameters is sampled to compute the angular distributions of the flow angles, $d N / d \cos \theta_{\mathrm{F}}$. The distribution of flow angles is computed by taking into account the formation of fragments via a six-dimensional coalescence model recently developed. ${ }^{8}$ We find roughly the same flow distribution by doing the flow analysis with and without clustering.

It should be pointed out that the intranuclear cascade model ${ }^{3,4}$ yields zero flow angles even at the highest multiplicities ${ }^{1,2,7,8}$ in contrast to the data. The present model, on the other hand, predicts peaks in the angular distributions of the flow angles. The peak shifts to larger angles with increasing multiplicity, in agreement with the data. The

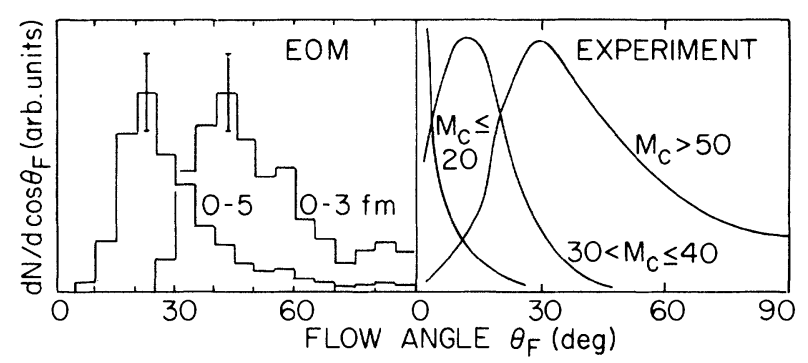

FIG. 2. Comparison of the experimental flow-angle distributions with the present theory, averaged over $b=0-3 \mathrm{fm}$ and $b=0-5 \mathrm{fm}$, respectively.

physical difference between the INC model and the EOM approach, which leads to such distinct predictions, can be traced back to the different treatments of the $n n$ collision process. The INC applies to a stochastic $4 \pi$ scattering at the point of closest approach of straight-line trajectories; this allows for substantial transparency. In contrast, the repulsive short-range component in the $N N$ potential used for the EOM approach is a hard core and thus effectively results in an excluded-volume effect; thus the nuclei are not as transparent and easily compressible as in the INC. This causes incident nucleons to be deflected away from zones of high density, i.e., small interparticle separations, towards sidewards angles. It is important to point out the role of the binding potential: The potential field keeps the nuclei from expanding before collisions can occur. If binding is neglected as in Cugnon's original program, ${ }^{4}$ finite flow angles occur because of sidewards expansion of the unbound projectile and target nucleons due to Fermi motion. ${ }^{8}$

Much more work remains before one can make a precise connection between the nuclear forces and the experiments on collective flow. Both the kinetic- and potential-energy contributions to the pressure tensor will be important, as will also the transport properties. It is a challenge for the EOM approach to make "realistic" calculations which accurately incorporate these and other requirements, such as spin and Pauli and uncertainty principles, as well as relativity, into the calculations.

(a) Permanent address: Laboratory for Atmospheric and Space Physics, University of Colorado, Boulder, Colo. 80309.

(b) Permanent address: TELCO Research Corporation, Nashville, Tenn. 37240.

${ }^{1}$ H. A. Gustafsson, H. H. Gutbrod, B. Kolb, H. Löhner, B. Ludewigt, A. M. Poskanzer, T. Renner, H. Riedesel, H. G. Ritter, A. Warwick, F. Weik, and 
H. Wieman, Phys. Rev. Lett. 52, 18 (1984).

${ }^{2}$ G. Buchwald, G. Graebner, J. Theis, J. Maruhn, W. Greiner, and H. Stöcker, Phys. Rev. Lett. 52, 18 (1984).

${ }^{3}$ Y. Yariv and Z. Frankel, Phys. Rev. C 20, 2227 (1979).

4J. Cugnon, D. Kinet, and J. Van der Meulen, Nucl. Phys. A379, 553 (1982).

${ }^{5}$ A. R. Bodmer and C. N. Panos, Phys. Rev. C 15, 1342 (1977). Transverse peaking (i.e., the $90^{\circ}$ cross section is larger than the $0^{\circ}$ cross section) has been observed in classical EOM calculations for central $(b=0)$ collisions of $A_{P}=A_{T}=40$ at $400 \mathrm{MeV} / \mathrm{u}$, with lighter systems being forward peaked: A. R. Bodmer, C. N. Panos, and A. D. MacKellar, Phys. Rev. C 22, 1025 (1980); A. R. Bodmer and C. N. Panos, Nucl. Phys. A356, 517 (1981). A detailed discussion of the transverse peaking and the role of the repulsive core and transparency effects can be found in A. R. Bodmer, in Proceedings of the Fifth Heavy-Ion Conference, University of California at Berkeley, Berkeley, California, 1981 (Univ. of California Press, Berkeley, 1981), p. 648.

${ }^{6}$ L. Wilets, E. M. Henley, M. Kraft, and A. D. MacKellar, Nucl. Phys. A282, 341 (1977); L. Wilets, Y. Yariv, and R. Chestnut, Nucl. Phys. A301, 359 (1978); D. J. E. Callaway, L. Wilets, and Y. Yariv, Nucl. Phys. A327, 250 (1979).

${ }^{7}$ M. Gyulassy, K. Frankel, and H. Stöcker, Phys. Lett. 110B, 185 (1982).

${ }^{8} \mathrm{H}$. Kruse, B. Jacak, and H. Stöcker, to be published. 